technique (the cDNA probes are not pure; certain messages may not be efficiently translated in vitro) make it difficult to trace the expression of any particular gene from transcription to translation with any confidence. An increase in experimental resolution will be required to distinguish the relative contributions of transcription and message stability to the rate of synthesis of specific proteins. A further criticism concerns the validity of the teratocarcinoma cell line as a model for normal muscle development. Although most of the identified muscle-specific polypeptides produced by the myotubes were characteristic of fast skeletal muscle, the authors also noted production of embryonic myosin light chain and slow muscle troponin $\mathrm{C}$, which implies that there are aberrations in the regulatory mechanisms of these cells. However, it is to be hoped that the experimental approach outlined in these papers will lay the foundations for a more detailed understanding of the mechanisms of myogenesis.

\title{
Multiphoton ionization interference effects
}

\section{from Peter Knight}

DIFFERENCES between results and theory in multiphoton ionization experiments seem to have been resolved by a recent experiment of the Saclay group (Morellec et al. Phys Rev. Lett. 44, 1394; 1980).

The ionization of atoms by the simultaneous absorption of several photons from an intense laser field has been the subject of intensive investigation recently, spurred on in part by the potential application to isotope separation. However, experiments and theory have been disagreeing by up to four orders of magnitude (Nature 270, 561; 1977) even for the simplest example of non-resonant twophoton ionization of a simple alkali such as caesium. Calculations employing secondorder perturbation theory all agree in predicting a deep interference minimum in the two-photon ionization cross-section of caesium at photon energies between 2.3 and $2.6 \mathrm{eV}$ arising from cancellations of $6 \mathrm{p}$ and $7 p$ intermediate state contributions. Experiments of Van der Wiel and colleagues ( $J$. Phys. B 8, 1617; 1975) saw no such minimum, and their observed crosssections were four orders of magnitude greater than expected from theory. Several attempts have since been made to explain this anomaly and the experiment of Morellec et al. seem to have resolved the problem by using a very much more intense laser than that of Van der Wiel.

Most workers in the field have suspected that an explanation of the discrepancy between theory and experiment was connected with the presence of a small number of alkali dimer molecules in the atomic beam target. To find the interference minimum is difficult experimentally: the atomic cross-section is (obviously) very small. On the other hand caesium molecules can ionize and produce a detector signal very easily in the

Peter Knight is in the Optics Section, Blackett Laboratory, Imperial College, London. wavelength range where the predicted atomic minimum should occur, since they possess many intermediate levels at the appropriate energies and can be reasonantly ionized. The molecules are in fact so easy to ionize that they can dominate the ionization rate even if their concentration is very low (a few percent) of the atomic concentration. Van der Wiel and his coworker did attempt to isolate and eliminate molecular contributions yet still found their anomalously high crosssections survived.

The Saclay group have now attacked the dimer problem in two ways. First, they work at much higher intensities than Van der Wiel using a three-stage oscillator-

Two-photon ionization cross-section of Cs atoms as a function of photon energy and laser wavelength (from Morellec et al. Phys Rev. Lett. 44, 1396; 1980). Triangles: experimental points of Van der Wiel normalized to new photoionization cross-sections; circles: experimental points of Morellec et al.; curves $a$, $b$ and $c$ are perturbative calculations noted in the text; curve $d$ is a theoretical result incorporating laser bandwidths in a simple model of

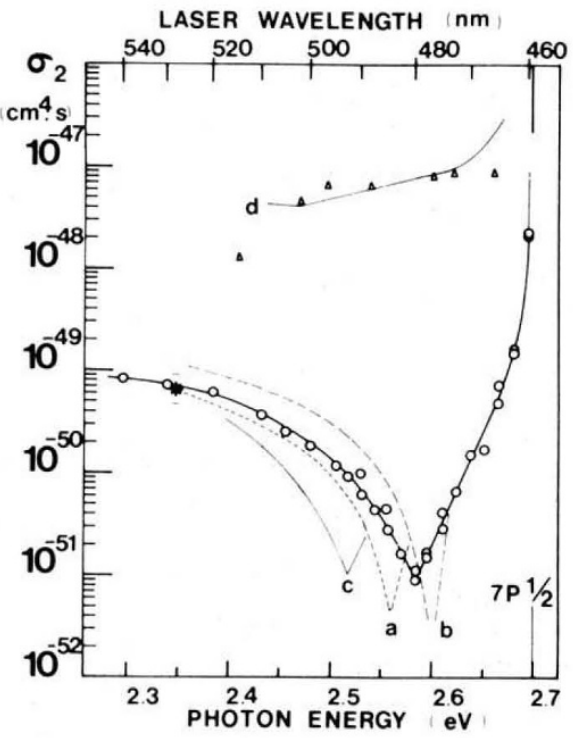

amplifier dye laser pumped by the secondharmonic of a ruby laser. Their experiments could then be carried out using laser intensities of $10^{10} \mathrm{~W} / \mathrm{cm}^{2}$ compared with $10^{5} \mathrm{~W} / \mathrm{cm}^{2}$ of Van der Wiel. Under such circumstances the resonant molecular ionization signal is saturated and is proportional to the intensity whereas the unsaturated atomic signal depends quadratically on the laser intensity and is easily distinguished from the molecular background. Secondly, the Saclay group performed their experiment with and without a caesium atomic beam superheater which efficiently dissociates dimers, and clearly see a reduction in the molecular component by more than an order of magnitude when the superheater is used. Using these two improvements together results in the atomic signal dominating the unwanted molecular signal in the $10-20 \mathrm{GW} / \mathrm{cm}^{2}$ laser intensity range.

The Saclay results for the ionization count are converted into a value for the two-photon generalized cross-section $\sigma_{2}$ taking into account well-known multimode coherence effects. Their results for $\mathrm{O}_{2}$ are shown in the figure and compared with earlier results of Van der Wiel and various theroetical values. It is clear that the expected ionization minimum has been observed, with a reduction by two orders of magnitude in $\sigma_{2}$ in going from photon energies of 2.3 to $2.58 \mathrm{eV}$, and then a huge increase as the photon energy approaches the $7 p_{1 / 2}$ intermediate-state resonance. Curves $a, b$ and $c$ are all derived from second-order perturbation theory using different atomic calculational methods: curve $a$ is due to Bebb (Phys. Rev. 149, 26; 1966), curve $b$ to Teague et al. (Phys. Rev. A14, 1057; 1976) and $c$ to Rachman et al. (Phys. Lett. 68A, 433; 1978). These second-order perturbative theories are in quite good agreement with each other and with the Saclay data, and disagree with the data of Van der Wiel et al. Curve $d$ in the figure, due to Armstrong and Eberly ( $J$. Phys. B12, L291; 1979), although agreeing with Van der Wiel's data, disagrees with the new results and the second-order theoretical results. Curve $d$ is obtained by incorporating laser fluctuations into the ionization dynamics in a first approximation using a phase-diffusion model of the laser bandwidth. This clearly exaggerates the laser bandwidth in the wings of the laser line and over-emphasises fluctuations in a way anticipated by Armstrong and Eberly who are currently working on improved laser-bandwidth models. The conclusion is that for the system studied by the Saclay group, second-order perturbation theory is adequate, that the interference minimum is observed once precautions are taken to eliminate molecular background, and that the earlier experiments at lower intensity were probably limited by this molecular background. It looks as if the non-resonant multiphoton ionization of simple atoms is now fairly well understood. 